\title{
Doping properties of $\mathrm{C}$, $\mathrm{Si}$, and Ge impurities in GaN and $\mathrm{AIN}$
}

\author{
P. Bogusławski \\ Department of Physics, North Carolina State University, Raleigh, North Carolina 27695 \\ and Institute of Physics, PAN, 02-668 Warsaw, Poland \\ J. Bernholc \\ Department of Physics, North Carolina State University, Raleigh, North Carolina 27695
}

(Received 9 May 1997; revised manuscript received 8 July 1997)

\begin{abstract}
Doping properties of substitutional $\mathrm{C}$, Si, and Ge impurities in wurtzite GaN and AlN were studied by quantum molecular dynamics. We considered incorporation of impurities on both cation and anion sublattices. When substituting for cations, $\mathrm{Si}$ and Ge are shallow donors in GaN, while Ge becomes a deep donor in AlN. Both impurities are deep acceptors on the $\mathrm{N}$ site. Substitutional $\mathrm{C}_{\text {cation }}$ is a shallow donor in $\mathrm{GaN}$, but a deep one in AlN; $\mathrm{C}_{\mathrm{N}}$ is a relatively shallow acceptor in both materials. Two effects that potentially quench doping efficiency were investigated. The first one is the transition of a donor from a substitutional position to a $D X$-like configuration. In crystals with a wurtzite symmetry, there are two possible variants of a $D X$-like state, and they have substantially different properties. In $\mathrm{GaN}, D X^{-}$states of both $\mathrm{Si}$ and $\mathrm{Ge}$ are unstable, or metastable, and thus they do not affect doping efficiency. In contrast, they are stable in AlN, and therefore neither $\mathrm{Si}$ nor $\mathrm{Ge}$ is a dopant in this material. Estimates obtained for $\mathrm{Al}_{x} \mathrm{Ga}_{1-x} \mathrm{~N}$ alloys show that the crossover composition for $D X$ stability is much lower for $\mathrm{Ge}(x \simeq 0.3)$ than for $\mathrm{Si}(x \simeq 0.6)$. The second effect quenching the doping efficiency is self-compensation, i.e., simultaneous incorporation of impurity atoms on both cation and anion sublattice. This effect may be enhanced by the formation of nearest-neighbor donor-acceptor pairs. The calculated binding energies of such pairs are large, about $1 \mathrm{eV}$, influencing self-compensation in some cases. Finally, the computed formation energies are used to identify growth conditions under which all these impurities may be efficient dopants in wide-band-gap nitrides. [S0163-1829(97)02340-0]
\end{abstract}

\section{INTRODUCTION}

Wide-band-gap nitrides are of considerable interest due to applications in blue/UV light-emitting diodes and lasers, and in high-temperature electronics. ${ }^{1,2}$ To exploit fully the potential of these materials, understanding and control of doping needs to be achieved. In the present work we study substitutional $X=\mathrm{C}, \mathrm{Si}$, and $\mathrm{Ge}$ impurities in hexagonal (wurtzite) $\mathrm{GaN}$ and AlN. This choice is motivated by the frequent usage of these species as dopants of III-V semiconductors. ${ }^{3-9}$ Further, both C (Ref. 10) and Si may be unintentionally incorporated as contaminants during growth.

In general, doping properties of group-IV atoms in a III-V compound are much more complex than those of group-II or VI atoms. This is because in the two latter cases the doping efficiency is determined by the electronic structure of the dopant, and limited only by its solubility. However, a group-IV atom is likely to become a donor when incorporated on the cation site, and an acceptor on the anion site. Thus a problem inherent to doping with group-IV elements is self-compensation, i.e., simultaneous incorporation of the dopant on both cation and anion sublattices. In GaN and AlN compounds, where there are large differences between the atomic radii of cations and anions, one could expect that self-compensation is blocked by strain effects. For example, a carbon atom in $\mathrm{GaN}$ should substitute for nitrogen, since the two atoms have similar atomic radii, while the substitution for the much larger gallium induces a large lattice strain energy of a few eV. However, in competition with the strain effects are processes of electron transfer from donors to ac- ceptors. Due to the wide band gap of nitrides, they lead to large energy gains, thereby enhancing self-compensation. The tendency toward self-compensation is further increased by the formation of donor-acceptor pairs. According to our results, binding energies of nearest-neighbor donor-acceptor pairs are about $1 \mathrm{eV}$, due to both the Coulomb coupling and an additional short-range interaction of comparable strength.

Another factor which may affect the doping is a transition of $X_{\text {cation }}$ donors from a substitutional to a $D X$-like state. In the wurtzite structure there are two nonequivalent $D X$-like configurations, in which the broken bond between the impurity and the host atom is either parallel or largely perpendicular to the $c$ axis. We have analyzed both variants and found that their properties are unexpectedly different in terms of stability and electronic structure. The transformation of the impurity to a $D X$ configuration is commonly accompanied by a capture of a second electron by the donor, which quenches the doping efficiency. According to our results, this process does not occur for either $\mathrm{Si}$ or $\mathrm{Ge}$ in $\mathrm{GaN}$ (and indeed the quenching of the doping is not observed). In AlN, however, the $D X^{-}$states are stable for both $\mathrm{Si}$ and $\mathrm{Ge}$, implying that neither impurity is a dopant.

The present paper considers all of the above issues. This allows us to identify the conditions of growth under which $\mathrm{C}$, $\mathrm{Si}$, and $\mathrm{Ge}$ are excellent dopants, as well as the conditions for which substantial quenching of the doping efficiency should be expected. In addition, the studies of doping of pure $\mathrm{GaN}$ and $\mathrm{AlN}$ are extended to those of $\mathrm{Al}_{x} \mathrm{Ga}_{1-x} \mathrm{~N}$ alloys. We find that these properties are strongly dependent on the alloy composition. 

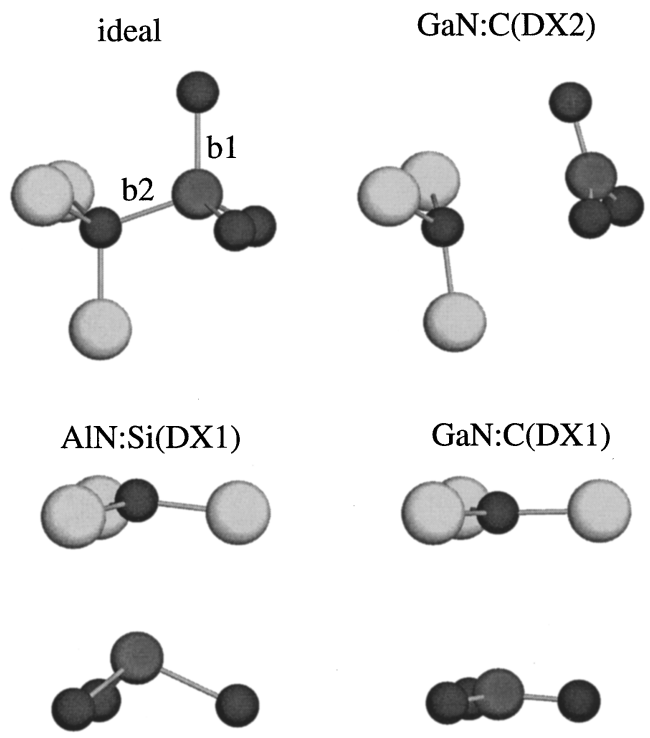

FIG. 1. Atomic configurations for the non-relaxed substitutional impurity, and for $D X$ states of $\mathrm{C}$ and $\mathrm{Si}$. Light gray spheres, $\mathrm{Ga}$; small black, $\mathrm{N}$; and medium gray, impurity atoms.

\section{METHOD OF CALCULATIONS}

The calculations were performed using quantum molecular dynamics, ${ }^{11}$ with atoms being efficiently relaxed using a special friction force. ${ }^{12}$ Soft pseudopotentials for $\mathrm{N}$ and $\mathrm{C}$ were used, ${ }^{13}$ which allowed for a relatively low plane-wave cutoff of $30 \mathrm{Ry}$. The pseudopotential of Ge was generated according to Ref. 14. Impurities were placed in a 72-atom supercell. Due to the size of the cell, the summations over the Brillouin zone were approximated by one $k$ point $(\Gamma)$. The $d$ electrons of $\mathrm{Ga}$ were treated as core states. This approximation leads to errors of about $0.2 \mathrm{eV}$ in the band gap and $0.3 \mathrm{eV}$ in the cohesive energy of zinc-blende GaN. ${ }^{15} \mathrm{In}$ the calculations, nonlocal core corrections were neglected. Our very recent results on native defects in $\mathrm{Al}_{x} \mathrm{Ga}_{1-x} \mathrm{~N}$ alloys, obtained using the potentials of Ref. 16, show that the formation energies computed with and without the nonlocal core corrections agree to within $0.2 \mathrm{eV}$. Further errors result from the use of the local-density approximation, which is well known to underestimate semiconductor band gaps, but these errors can be minimized by quoting the positions of the impurity-induced levels with respect to the nearest band edges. In particular, the effective-mass character found for several impurity-induced states is a feature that will persist in more accurate calculations.

\section{SUBSTITUTIONAL CONFIGURATIONS AND STRAIN EFFECTS}

We first discuss configurations of substitutional impurities and strain effects. A substitutional impurity in the crystal with wurtzite symmetry has four nearest neighbors. One of them, located along the $c$ axis relative to the impurity (here called a type-1 neighbor), is nonequivalent by symmetry to the remaining three neighbors (here called type-2 neighbors), see Fig. 1. For the group-IV impurities considered here, the nonequivalence effect is small, since the bond lengths with type- 1 and -2 neighbors differ by less than $1.5 \%$ in most
TABLE I. Effects of atomic relaxations around neutral substitutional impurities: $\Delta b$ is the change of the bond length, $E_{\text {rel }}$ is the relaxation energy, and $\epsilon_{\mathrm{imp}}$ is the position of the impurity level, with e.m. denoting effective-mass states.

\begin{tabular}{lccc}
\hline \hline & $\Delta b(\%)$ & $E_{\text {rel }}(\mathrm{eV})$ & $\epsilon_{\text {imp }}(\mathrm{eV})$ \\
& \multicolumn{4}{c}{$\mathrm{GaN}$} & \\
\hline $\mathrm{C}_{\mathrm{Ga}}$ & -18.1 & 1.65 & e.m. \\
$\mathrm{Si}_{\mathrm{Ga}}$ & -5.6 & 0.65 & e.m. \\
$\mathrm{Ge}_{\mathrm{Ga}}$ & -1.4 & 0.25 & e.m. \\
$\mathrm{C}_{\mathrm{N}}$ & -2.0 & 0.1 & $E_{v}+0.2$ \\
$\mathrm{Si}_{\mathrm{N}}$ & 13.6 & 3.9 & $E_{v}+1.2$ \\
$\mathrm{Ge}_{\mathrm{N}}$ & 13.5 & 4.1 & $E_{v}+1.35$ \\
& & $\mathrm{AlN}$ & \\
\hline $\mathrm{C}_{\mathrm{Al}}$ & -3.0 & unstable & \\
$\mathrm{Si}_{\mathrm{Al}}$ & 17.2 & 0.3 & e.m. \\
$\mathrm{Ge}_{\mathrm{Al}}$ & 2.0 & 0.9 & $E_{c}-1.0$ \\
$\mathrm{C}_{\mathrm{N}}$ & 17.5 & 0.9 & $E_{v}+0.5$ \\
$\mathrm{Si}_{\mathrm{N}}$ & 17.5 & 6.9 & $E_{v}+2.0$ \\
$\mathrm{Ge}_{\mathrm{N}}$ & & 7.4 & $E_{v}+2.1$ \\
\hline \hline
\end{tabular}

cases (the exceptions, $\mathrm{C}_{\mathrm{Al}}$ and $\mathrm{Ge}_{\mathrm{Al}}$, are discussed below). In all cases we find breathing-mode distortions that preserve the local hexagonal symmetry. The calculated changes of bond lengths relative to the bulk values (which are $b_{1}=$ $b_{2}=1.96 \AA$ for GaN, and $b_{1}=1.93$ and $b_{2}=1.89 \AA$ for $\mathrm{AlN}$ ) are given in Table I. We also list energy gains $E_{\text {rel }}$ due to the relaxation from the ideal substitutional configuration to the final one. As is evident from the table, the inclusion of relaxation effects is in general necessary for a proper description of impurities in nitrides. In cases when the mismatch between the atomic radii of impurity and the host atom is large, the calculated relaxation energies are one order of magnitude greater than those found in typical III-V compounds like GaAs. The most drastic case is that of $\mathrm{Ge}_{\mathrm{N}}$ in AlN; lattice relaxation releases an elastic energy of about 7 $\mathrm{eV}$, increases the length of $\mathrm{Ge}-\mathrm{N}$ bonds by $17 \%$, and rises the acceptor level by $1.3 \mathrm{eV}$. Very similar results are obtained for $\mathrm{AlN}: \mathrm{Si}_{\mathrm{N}}$. The calculated $\mathrm{E}_{\text {rel }}$ are systematically greater for AlN than for GaN, reflecting the greater stiffness of AlN. Finally, we observe that even after the relaxation, i.e., at equilibrium, the bonds around impurity remain strained (or stretched), which induces a residual strain energy. Simple arguments and estimates based on the valence force field model ${ }^{17}$ show that the residual strain energy is comparable to the relaxation energy $E_{\text {rel }}$. In other words, only one-half of the initial strain is released during the relaxation. Large values of the residual strain suggest that formation of impuritynative defect complexes could be favored energetically. The implications of this effect for the stability of $D X$ states are discussed in Sec. IV G.

\section{STRUCTURE OF DONORS}

In this section we consider the properties and electronic structure of $\mathrm{C}, \mathrm{Si}$, and $\mathrm{Ge}$, substituting for the cation and becoming donors in both GaN and AlN. The discussion of results is complicated by the fact that, in many cases, the ground-state configuration depends on the charge state of the 


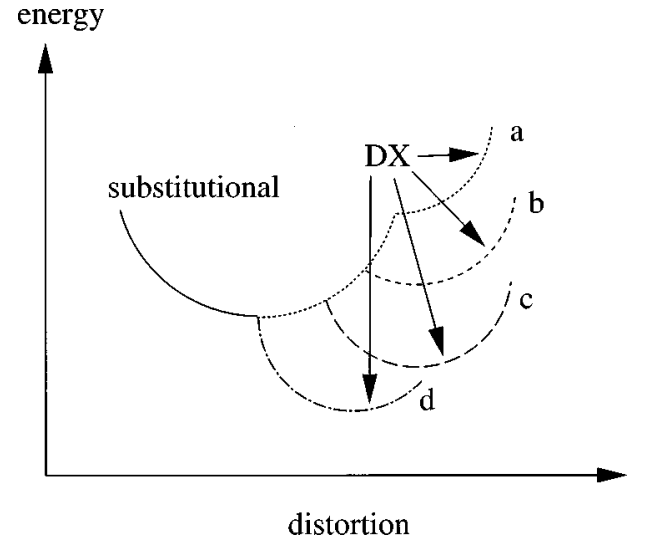

FIG. 2. Schematic configuration diagram of an impurity, comparing energies of substitutional and $D X$-like states: (a) the $D X$ state is unstable; in (b) and (c) both substitutional and $D X$ states are local minima separated by an energy barrier; and in (d) the substitutional configuration unstable.

impurity. We analyze two possible atomic configurations of a donor. The first one is substitutional, with almost equal bonds with its nearest neighbors. The second configuration is of $D X$ type $^{18}$ In this case, a bond between the impurity and one of its first neighbors is broken, and one (or both) of these atoms move from substitutional sites to interstitial locations. Figure 2 shows a schematic configuration diagram of an impurity, which takes into account both states, i.e., the substitutional one and the $D X$-like state with a large lattice distortion. Case (a) corresponds to the situation when the $D X$ state is unstable, and the impurity comes back to the substitutional state without an energy barrier. In case (b), the $D X$ state is higher in energy than the substitutional state, but there is an energy barrier between the two states and the $D X$ configuration is metastable. Case (c) is similar to case (b), but the energy of the $D X$ state is lower than that of the substitutional configuration. Finally, in case (d) the substitutional site is unstable, and the transformation to the $D X$ state occurs with no energy barrier. As will be discussed in the following, all possible situations (a)-(d) are found for the group-IV impurities in the nitrides.

An additional complication comes from the fact that in the wurtzite structure there are two nonequivalent $D X$ configurations, for which the broken bond is between the impurity and either the type- 1 or type- 2 neighbor. They are referred to as $D X_{1}$ and $D X_{2}$ variants in the following. We have considered both cases and found that their properties differ substantially. In particular, the $D X_{1}$ variant is less stable than $D X_{2}$ for $\mathrm{C}^{-}$, and more stable for $\mathrm{Si}^{-}$and $\mathrm{Ge}^{-}$. The properties of the impurities are qualitatively different in $\mathrm{GaN}$ and AlN, which is due in part to the wider band gap of the latter. Consequently, the impurity states in AlN are in general deeper and more localized, and the tendency to stabilize the $D X$-type geometry is more pronounced. ${ }^{19}$ The results are summarized in Table II.

\section{A. GaN:C}

In the case of substitutional $\mathrm{C}$ in $\mathrm{GaN}$, the $\mathrm{C}$-derived level is a resonance situated at $0.8 \mathrm{eV}$ above the bottom of the conduction band. Its position rises by $1.2 \mathrm{eV}$ due to relax-
TABLE II. Energies in eV of substitutional (denoted by subst.) impurities on the cation site, and of $D X$ configurations relative to those of the ground state (GS); and distances between the impurity and the host $\mathrm{N}^{*}$ atom. Unstable configurations are denoted by unst. See text for notation.

\begin{tabular}{lcccccc}
\hline \hline Dopant & subst. & $b_{1}(\AA)$ & $\begin{array}{c}D X_{1} \\
\text { GaN }\end{array}$ & $b_{1}(\AA)$ & $D X_{2}$ & $b_{2}(\AA)$ \\
\hline $\mathrm{C}(+)$ & 0.05 & 1.62 & 0.65 & 3.05 & $\mathrm{GS}$ & 2.85 \\
$\mathrm{C}(0)$ & 0.05 & 1.63 & 0.60 & 3.25 & $\mathrm{GS}$ & 2.96 \\
$\mathrm{C}(-)$ & 0.1 & 1.62 & 0.60 & 3.31 & $\mathrm{GS}$ & 3.15 \\
$\mathrm{Si}(0)$ & $\mathrm{GS}$ & 1.85 & unst. & & unst. & \\
$\mathrm{Si}(-)$ & $\mathrm{GS}$ & 1.85 & unst. & & unst. & \\
$\mathrm{Ge}(0)$ & $\mathrm{GS}$ & 1.93 & unst. & & unst. & \\
$\mathrm{Ge}(-)$ & $\mathrm{GS}$ & 1.93 & 0.60 & 2.61 & unst. & \\
& & & & & & \\
& & & AlN & & & \\
\hline $\mathrm{C}(+)$ & GS & 1.62 & 0.15 & 2.91 & 0.30 & 2.89 \\
$\mathrm{C}(0)$ & unst. & & 0.05 & 2.97 & $\mathrm{GS}$ & 3.05 \\
$\mathrm{C}(-)$ & unst. & & 0.7 & 2.97 & $\mathrm{GS}$ & 3.38 \\
$\mathrm{Si}(+)$ & $\mathrm{GS}$ & 1.85 & & & & \\
$\mathrm{Si}(0)$ & GS & 1.87 & 0.3 & 2.82 & unst. & \\
$\mathrm{Si}(-)$ & 1.4 & 1.88 & GS & 2.98 & 0.95 & 2.93 \\
$\mathrm{Ge}(+)$ & GS & 2.07 & unst. & & unst. & \\
$\mathrm{Ge}(0)$ & GS & 2.26 & unst. & & unst. & \\
$\mathrm{Ge}(-)$ & unst. & & GS & 2.90 & unst. & \\
\hline \hline
\end{tabular}

ation. For the neutral charge state of the impurity, the electron that should occupy the resonance autoionizes to the conduction band, and becomes trapped on the shallow level by the Coulomb tail of the impurity potential. However, the $D X_{2}$ and the substitutional configurations are energetically almost degenerate. We find that the energy of the $D X_{2}$ configuration is lower by about $0.1 \mathrm{eV}$ than the substitutional one, but this small difference is within the accuracy of our calculations. The $D X_{1}$ variant $\mathrm{C}_{\mathrm{Ga}}^{D X_{1}}$ is metastable, with energy higher than that of $D X_{2}$ by about $0.6 \mathrm{eV}$ for all charge states. Therefore, the carbon impurity in $\mathrm{GaN}$ corresponds to cases (b) and/or (c) in Fig. 2, since the two energy minima have almost the same energy.

The atomic positions for the $D X$-like configurations are shown in Fig. 1. In the $D X_{2}$ state, both the host $\mathrm{N}$ atom (denoted here by $\mathrm{N}^{*}$ ) and the impurity are significantly displaced along the $c$ axis. The distance between both atoms is about $3 \AA$ in the neutral charge state, which is almost twice the nearest-neighbor distance $(1.62 \AA)$ in the substitutional geometry. The final positions of these atoms are close to the centers of "triangles" formed by their neighbors. As in the $D X_{2}$ configuration, in the $D X_{1}$ variant both atoms are displaced from the lattice sites, and approach the centers of triangles formed by their neighbors; the distance between the two atoms is again $\sim 3.2 \AA$. For both $D X$ geometries, the $\mathrm{C}-\mathrm{N}^{*}$ distance is the largest for the negative charge states, and the shortest for positive ones. Considering the electronic structure, we find that $\mathrm{C}^{D X_{1}}\left(\mathrm{C}^{D X_{2}}\right)$ introduces a singlet at about $0.3(0.6) \mathrm{eV}$ above the top of the valence band, and another singlet level at about $0.4(0.5) \mathrm{eV}$ below the bottom of the conduction band. Thus $\mathrm{C}_{\mathrm{Ga}}$ in $\mathrm{GaN}$ is a deep donor when in a $D X$ configuration. 


\section{B. GaN:Si}

$\mathrm{Si}_{\mathrm{Ga}}$ in $\mathrm{GaN}$ is a shallow effective-mass donor. According to our results, $\mathrm{Si}$ is unstable in both $D X$ variants, since there are no energy barriers for the transition from the initial $D X$-like configurations, with large lattice distortions, to the substitutional ones. This holds for both the neutral and the negatively charged $\mathrm{Si}$. Thus $\mathrm{Si}_{\mathrm{Ga}}^{-}$is described by case (a) in Fig. 2.

\section{GaN:Ge}

$\mathrm{Ge}_{\mathrm{Ga}}$, like $\mathrm{Si}_{\mathrm{Ga}}$, is a shallow effective-mass donor in $\mathrm{GaN}$. Neither Si nor Ge introduce a resonance for energies up to $1.5 \mathrm{eV}$ above the bottom of the conduction band. However, unlike $\mathrm{Si}$, the negatively charged $\mathrm{Ge}$ is metastable in the $D X_{1}$ state, and its energy is higher by $0.6 \mathrm{eV}$ than that of substitutional $\mathrm{Ge}^{-}$[case (b) in Fig. 2]. For the neutral charge state, the $D X_{1}$ configuration is unstable, and the impurity comes back to the substitutional site with no energy barrier [case (a) in Fig. 2]. We also find that the $D X_{2}$ variant is unstable for both the neutral and negative charge states. The geometry of the $D X_{1}$ state is different than that obtained for $\mathrm{C}_{\mathrm{Ga}}^{D X}$. The Ge atom is essentially located at the lattice site, and only the nearest-neighbor $\mathrm{N}^{*}$ atom is displaced along the $c$ axis. The Ge-N* distance is $2.61 \AA$, to be compared with $1.93 \AA$ for the substitutional configuration. $\mathrm{Ge}_{\mathrm{Ga}}^{D X_{1}}(-)$ introduces a singlet, almost degenerate with the valenceband top, and a second singlet about $0.5 \mathrm{eV}$ below the bottom of the conduction band.

\section{AIN:C}

The ground-state configuration of the carbon impurity in AlN depends on its charge state. For $\mathrm{C}_{\mathrm{Al}}$ in the positive charge state, the ground state is the substitutional configuration, while the $D X_{1}$ and $D X_{2}$ variants are higher in energy by 0.15 and $0.3 \mathrm{eV}$, respectively. Similarly to $\mathrm{GaN}: \mathrm{C}$, for the neutral and the negative charge states the $D X_{2}$ variant is the ground state, and its energy is lower that of $D X_{1}$ by 0.1 and $0.7 \mathrm{eV}$, respectively. Furthermore, for both the neutral and the negative charge states the substitutional configurations are unstable, and transform to the $D X_{1}$ states. This situation is schematically shown as case (d) in Fig. 2. The atomic configurations of $\mathrm{C}_{\mathrm{Al}}^{D X}$ in both $D X$ variants are similar to $\mathrm{C}_{\mathrm{Ga}}^{D X}$ described above and shown in Fig. 1; both $\mathrm{C}$ and $\mathrm{N}^{*}$ move along the axis of the broken bond, and occupy positions close to the center of triangles of their respective neighbors. The distance between them increases to about $3.0 \AA$ (depending on the charge state), which is almost two times larger than $1.62 \AA$ found for the substitutional case.

$\mathrm{C}_{\mathrm{Al}}^{D X_{1}}(-)$ introduces a singlet at about $0.3 \mathrm{eV}$ above the top of the valence band, and a singlet about $1.7 \mathrm{eV}$ below the bottom of the conduction band. The levels introduced by $\mathrm{C}_{\mathrm{Al}}^{D X_{2}}$ are deeper, at about $0.9 \mathrm{eV}$ above the valence band top, and at about $1.6 \mathrm{eV}$ (for $D X_{2}^{+}$and $D X_{2}^{0}$ ) and $2.7 \mathrm{eV}$ (for $D X_{2}^{-}$) below the bottom of the conduction band.

\section{E. AIN:Si}

The substitutional configuration is the ground state for both the positive and neutral charge states of $\mathrm{Si}_{\mathrm{Al}}$. In this configuration, $\mathrm{Si}$ is an effective mass donor. The $D X_{1}(0)$ variant is higher by $0.3 \mathrm{eV}$ than the ground state, and the $D X_{2}(0)$ variant is unstable. However, for the negative charge state the ground state is the $D X_{1}$ variant, with the energy lower by $1.4 \mathrm{eV}$ than that of the substitutional case. The $D X_{2}(-)$ state is metastable, and its energy is higher by 0.95 $\mathrm{eV}$ than that of $D X_{1}(-)$. The atomic configuration of the $D X_{1}$ variant for $\mathrm{Si}_{\mathrm{Al}}$ in $\mathrm{AlN}$ is shown in Fig. 1. In this variant, the impurity remains on the substitutional site, the $\mathrm{N}^{*}$ atom is strongly displaced above the triangle of its $\mathrm{Ga}$ neighbors, and the impurity-host distance increases to $3.0 \AA$, compared to $1.88 \AA$ in the substitutional case. In the $D X_{2}(-)$ variant, both atoms are displaced from the ideal lattice sites, and the $\mathrm{Si}-\mathrm{N} *$ distance is $2.93 \AA$. Si in the $D X_{1}(-)$ variant introduces a level at about $0.2 \mathrm{eV}$ above the valence band top, and at about $-1.9 \mathrm{eV}$ below the bottom of the conduction band.

\section{F. AlN:Ge}

$\mathrm{Ge}_{\mathrm{Al}}(0)$ is a deep donor, with the energy level located at about $1 \mathrm{eV}$ below the bottom of the conduction band. The localization of the wave function on $\mathrm{Ge}$ is accompanied by a large distortion of the atomic configuration around this donor; the nearest $\mathrm{N}$ neighbors relax outwards increasing the $b_{1}$ bond by $17 \%$, and $b_{2}$ by $10 \%$ relative to the bulk values. This contrasts with the small relaxation found for $\mathrm{Ge}_{\mathrm{Ga}}$ which is a shallow donor. For the neutral charge state, both $D X$ variants are unstable. However, in the negative charge state $\mathrm{Ge}$ relaxes from the substitutional site towards the $D X_{1}$ configuration. The $D X_{2}(-)$ variant is unstable. The atomic configuration of the $D X_{1}$ variant for $\mathrm{Ge}_{\mathrm{Al}}$ is similar to this for $\mathrm{Si}_{\mathrm{Al}}^{D X_{1}}$ in AlN shown in Fig. 1 . In the $D X_{1}$ state the Ge atom occupies the substitutional site and the $\mathrm{N}^{*}$ atom is displaced. Ge in the $D X_{1}(-)$ variant introduces a level at about $0.2 \mathrm{eV}$ above the valence-band top, and a very deep level at about $-2.4 \mathrm{eV}$ below the bottom of the conduction band. Finally, the calculated equilibrium configuration of $\mathrm{Ge}_{\mathrm{Al}}(0)$ closely resembles the $D^{-}$states in III-V zinc-blende semiconductors with large breathing-mode relaxations. ${ }^{20}$ Considering the issue of $D^{-}$, we observe that a state with a large breathing mode distortion is a stable configuration of Ge in AlN, but in the neutral charge state. However, in the negative charge state this configuration is unstable, and the impurity transforms to a $D X$ state. In GaAs, the $D^{-}$state is more stable for $\mathrm{Ge}$ than for $\mathrm{Si}^{20}{ }^{20}$ we have thus not analyzed this state for $\mathrm{Si}$.

From the results of Secs. IV A-IV F, it follows that in all cases considered here, the breaking of an impurity-host bond leads to the formation of two levels in the band gap. To analyze structures of defect-induced levels we have projected their wave functions onto atomic orbitals. ${ }^{21}$ The first level, denoted here by $L_{1}$, is energetically close to the top of the valence band. It is occupied by two electrons for all charge states of the impurity. It is localized on the displaced $\mathrm{N}^{*}$ atom, although the degree of localization strongly depends on the system, displaying the ability of valence electrons to screen the broken bond. The second, higher level, denoted here by $L_{2}$, is pulled down from the conduction bands to the band gap. The $L_{2}$ level of the $D X_{1}$ variant is composed of comparable amounts of the $s$ and $p_{z}$ orbitals of the impurity, 
the $s p^{3}$ orbitals of the three $\mathrm{N}$ nearest neighbors of the impurity, and the $p_{z}$ orbital of $\mathrm{N}^{*}$. In the $D X_{2}$ variant, the wave function of $L_{2}$ is composed from the $s$ and $p$ orbitals of the impurity, and of the triangle of $\mathrm{N}$ atoms around the impurity. In contrast to $D X_{1}$, the dangling bond of $\mathrm{N}^{*}$ does not contribute to $L_{2}$.

The properties of $D X$ states of $\mathrm{Si}$ and Ge are different from those of $\mathrm{C}$. This difference may be traced back to the respective electronic structures of the impurities. In the case of $\mathrm{Si}$ or $\mathrm{Ge}$, the degree of localization of $L_{1}$ is weak, since only about $10-15 \%$ of the wave function is localized on $\mathrm{N}^{*}$. To complete the screening of broken bonds and stabilize the $D X$ configurations, two electrons must occupy the $L_{2}$ level (which is much more localized than $L_{1}$ ). In the case of $\mathrm{C}$, however, the $L_{1}$ level is highly localized. The contribution of $\mathrm{N}^{*}$ orbitals is $35 \%$ in the $D X_{1}$ variant, and two times greater in the more stable $D X_{2}$ variant. Thus broken bonds are screened by the $L_{1}$ state to a large extent, and the occupation of $L_{2}$ is not necessary to stabilize the $D X$ configurations.

Another factor adding to the stability of carbon in $D X$ configurations relative to the substitutional ones comes from strain effects. When $\mathrm{C}$ substitutes for the much larger $\mathrm{Ga}$ or $\mathrm{Al}$ atom, the $\mathrm{C}-\mathrm{N}$ bonds are highly stretched, because they are shorter than the equilibrium cation- $\mathrm{N}$ bonds by about $15 \%$. This induces a high excess strain energy even after relaxation, since the $\mathrm{C}-\mathrm{N}$ bonds remain stretched. The value of this excess elastic energy, estimated by the valence force field model,,${ }^{17}$ is about $3 \mathrm{eV}$. (Note that this value is comparable to the cohesive energy per bond in the host crystals.) However, in the $D X$ configurations one $\mathrm{C}-\mathrm{N}$ bond is broken, and both $\mathrm{C}$ and $\mathrm{N}^{*}$ are free to relax and release part of the excess strain by shortening the bonds with their neighbors. Consequently, the $\mathrm{C}_{\text {cation }}^{D X}-\mathrm{N}^{*}$ bonds are shorter by about $5 \%$ than the $\mathrm{C}_{\text {cation }}-\mathrm{N}$ bonds in the substitutional case. Moreover, $\mathrm{C}^{D X}$ forms a nearly planar configuration with its neighbors; see Fig. 1. This geometry is additionally stabilized by the tendency of $\mathrm{C}$ to form planar $s p^{2}$ bonds. We notice that both factors contribute to the stabilization of $\mathrm{C}_{\text {cation }}^{D X}$ in the neutral charge state, and even of the positive $\mathrm{C}_{\mathrm{Ga}}$.

\section{G. Stability of $D X$ states in $\mathrm{Al}_{x} \mathrm{Ga}_{1-x} \mathrm{~N}$ alloys}

The predicted stability of $D X^{-}$states for $\mathrm{Si}$ and $\mathrm{Ge}$ in AlN has important implications for the efficiency of $n$-type doping using these species. The stability of $D X^{-}$states implies that the reaction

$$
2 d^{0} \rightarrow d^{+}+D X^{-}
$$

is exothermic. In this case, the electrons are captured on deep impurity levels (i.e., on the $L_{2}$ states discussed above), and the doping does not result in a conducting sample. According to our results, this occurs for both $\mathrm{Si}$ and Ge in AlN. On the other hand, $\mathrm{Si}$ and $\mathrm{Ge}$ in $\mathrm{GaN}$ are excellent dopants since the $D X$ states are metastable or unstable, and reaction (1) is endothermic. These species are thus shallow donors. These results imply that the doping efficiency of both $\mathrm{Si}$ and $\mathrm{Ge}$ in $\mathrm{Al}_{x} \mathrm{Ga}_{1-x} \mathrm{~N}$ alloys should strongly depend on the alloy composition. In GaN-rich samples $\mathrm{Si}$ and $\mathrm{Ge}$ are efficient donors, while in the AlN-rich limit the doping efficiency is quenched. $^{22}$
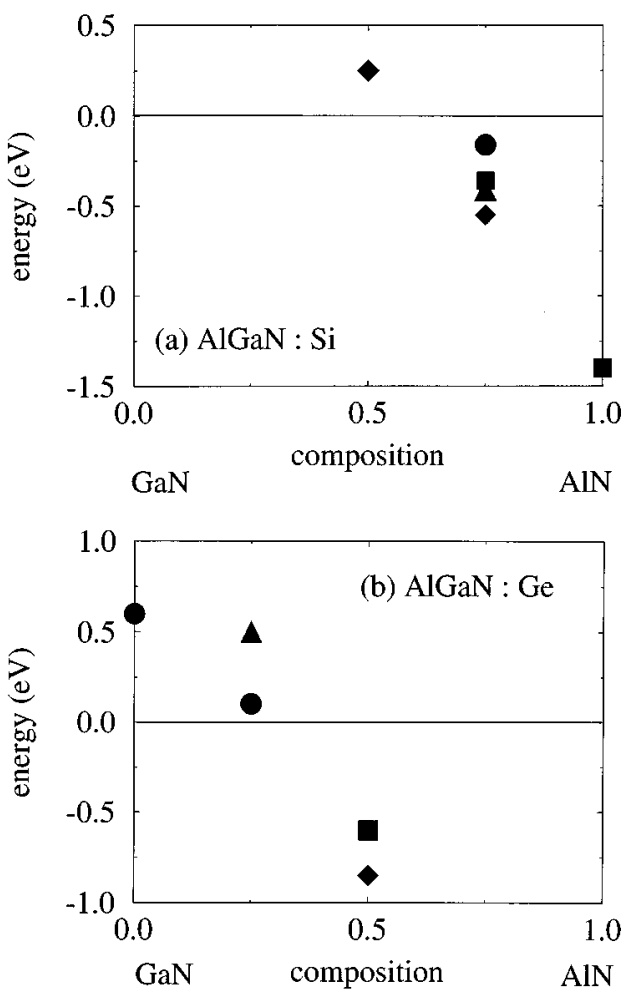

FIG. 3. Energies of $D X_{1}$ states of (a) $\mathrm{Si}$ and (b) Ge impurities in negative charge states relative to the substitutional configurations. Circles, diamonds, triangles, and squares denote the variants with three, two, one, and no Ga neighbors of $\mathrm{N}^{*}$.

To determine the crossover compositions above which the low-energy $D X_{1}$ variant is stable, we have performed calculations for $\mathrm{Al}_{x} \mathrm{Ga}_{1-} \mathrm{N}$ alloys with $x=0.25,0.5$, and 0.75 . The positions of the $\mathrm{Ga}$ and $\mathrm{Al}$ atoms in the supercell were chosen randomly. However, even in a random alloy, properties of a given impurity atom depend on the actual number and distribution of $\mathrm{Ga}$ and $\mathrm{Al}$ atoms in its surroundings, and in particular in the first two shells of its neighbors. This holds in particular for the relative stability of a $D X$ configuration compared to the substitutional case. In the case of $\mathrm{Si}$ or Ge substituting for a cation, the first neighbors of the impurity are $\mathrm{N}$ atoms. Therefore, since the chemical disorder occurs only in the second-neighbor (and more distant) shells of the impurity, we do not expect a substitutional donor to be very sensitive to the actual surroundings. However, the $D X_{1}$ states may be sensitive to the chemical disorder. This is because the displaced atom is not the impurity but the host $\mathrm{N}^{*}$ atom. In a $D X$ state, $\mathrm{N}^{*}$ is situated close to the center of a triangle of three cations, which are first neighbors of $\mathrm{N}^{*}$, and may be three $\mathrm{Ga}$ atoms, two $\mathrm{Ga}$ atoms and one $\mathrm{Al}$ atom, etc.

We have analyzed this effect for both Si and Ge by considering a few configurations of cations for a given alloy composition. The calculated results are shown in Fig. 3, where we compare energies of $D X_{1}$ states with those of the corresponding substitutional cases. ${ }^{23} \mathrm{We}$ see that the relative energy of the $D X$ state varies by about $0.4 \mathrm{eV}$ depending on the actual environment of the impurity. In most cases, lowering the number of $\mathrm{Ga}$ atoms in the triangle of first neighbors of $\mathrm{N}^{*}$ stabilizes the $D X$ state. The calculated magnitude of the alloy splitting of $D X$ states is somewhat higher than 
that measured for $\mathrm{Al}_{x} \mathrm{Ga}_{1-x} \mathrm{As}: \mathrm{Si}^{24}$ Interestingly, the impurity-induced $L_{1}$ and $L_{2}$ levels are less sensitive to the chemical disorder than the total energy, since they remain constant to within $\sim 0.05 \mathrm{eV}$ for a given alloy composition.

As a result of the chemical disorder, the transition from the Ga-rich alloys where the $D X$ states are metastable (or unstable), to the Al-rich alloys with the $D X$ states stable will not occur at a sharp crossover composition. Instead, one expects a finite composition range of the order of $10 \%$, over which the doping efficiency will gradually decrease. From Fig. 3 it follows that the crossover compositions of $\mathrm{Al}_{x} \mathrm{Ga}_{1-{ }_{x}} \mathrm{~N}$ are about 0.3 for $\mathrm{Ge}$, and about 0.6 for Si. Thus, from this point of view, $\mathrm{Si}$ is a donor superior to $\mathrm{Ge}$, since it remains an efficient dopant over a wider composition range.

\section{H. Comparison with experiment}

We will now compare our results for $\mathrm{C}, \mathrm{Si}$, and Ge impurities with the available experimental data. Recent investigations have shown that $\mathrm{C}$ is an acceptor slightly more shallow, by about $20 \mathrm{meV}$, than the commonly used $\mathrm{Mg}^{4}$ To date, a hole concentration of $\sim 3 \times 10^{17} \mathrm{~cm}^{-3}$ was obtained using doping with $\mathrm{CCl}_{4}$ in metallo-organic $\mathrm{MBE}$ (MOMBE) growth. ${ }^{6}$ Higher concentrations were not obtained since with the increasing $\mathrm{CCl}_{4}$ flow the growth rate is drastically reduced. One should note, however, that the theoretical solubility of $\mathrm{C}_{\mathrm{N}}$ is one order of magnitude higher.

Experimental investigations of $\mathrm{Si}$ in $\mathrm{GaN}$ were recently performed in Ref. 7. There it was shown that up to the concentrations of $4 \times 10^{19} \mathrm{~cm}^{-3}$, Si is incorporated without selfcompensation. The high-pressure study of Wetzel et al. ${ }^{8}$ showed that Si remains a shallow donor for pressures up to $27 \mathrm{GPa}$. This suggests an absence of a resonant state close to the bottom of the conduction band, and indicates that $\mathrm{Si}$ is a hydrogenic donor in $\mathrm{Al}_{x} \mathrm{Ga}_{1-x} \mathrm{~N}$ alloys with the composition range $0 \leqslant x \leqslant 0.3$. No conclusions for higher values of $x$ can be drawn. This also indicates that the possible $D X$ state is much higher in energy than in the case of GaAs, where the $D X$ state of $\mathrm{Si}$ is stable at pressures above $2 \mathrm{GPa}^{24}$ Bremser and Davis ${ }^{5}$ recently observed quenching of doping efficiency with $\mathrm{Si}$ for compositions higher than $60 \%$ which may be due to the stabilization of $D X$ states. Both experimental findings ${ }^{8,5}$ agree with our calculated properties of Si impurity.

Experimental investigations of the $\mathrm{Al}_{x} \mathrm{Ga}_{1-x} \mathrm{~N}$ :Ge were recently performed by Zhang et al. ${ }^{9}$ They found that Ge is an efficient dopant for $x \leqslant 0.2$, which covers the whole composition range of efficient doping with Ge predicted here. No data for higher $\mathrm{Al}$ content were given.

We end this section with two comments. First, we observe that well-known fingerprints of the (meta)stability of $D X$ states are the so-called persistent effects, e.g., persistent changes in conductivity induced by illumination of a sample cooled in darkness. They occur when both the substitutional and the $D X$ configurations of a negatively charged impurity are local minima separated by an energy barrier, which allows for a metastable, nonequilibrium distribution of electrons between the two states to exist. This corresponds to cases (b) and (c) in Fig. 2. One should observe that the conditions for the persistent effects can be different from those leading to quenching of the doping efficiency, which takes place in situations (c) and (d) in Fig. 2. Thus the two effects take place in different regimes of alloy composition. According to our results, two minima exist for $\mathrm{C}, \mathrm{Si}$, and $\mathrm{Ge}$ in $\mathrm{Al}_{x} \mathrm{Ga}_{1-x} \mathrm{~N}$ alloys with appropriate compositions, which may be estimated from Table II and Fig. 3. In particular, persistent effects for Ge and C may only occur in the Ga-rich regime, since in AlN the substitutional configurations are unstable, and both impurities undergo a transformation to the $D X_{1}$ variant without an energy barrier. $\mathrm{Si}^{-}$in $\mathrm{GaN}$ and $\mathrm{Ga}-$ rich $\mathrm{Al}_{x} \mathrm{Ga}_{1-x} \mathrm{~N}$ alloys is unstable in the $D X$ state, and thus persistent effects may only occur for $\mathrm{Al}$ contents higher than about 25\%. To our knowledge, such effects were not reported for $\mathrm{Al}_{x} \mathrm{Ga}_{1-x} \mathrm{~N}$ alloys for the dopants considered here.

The second comment concerns shallow effective mass donors in $\mathrm{Al}_{x} \mathrm{Ga}_{1-x} \mathrm{~N}$ alloys. The doping efficiency of substitutional $\mathrm{Si}$ and $\mathrm{Ge}$ decreases with an increasing $\mathrm{Al}$ content because, as was observed experimentally, the effective-mass states become progressively deeper with the increasing alloy composition due to both the increasing effective mass and the decreasing dielectric constant. We stress that this effect is independent of the stabilization of the $D X$ states with the increasing $\mathrm{Al}$ content.

\section{ELECTRONIC STRUCTURE OF ACCEPTORS}

We shall now consider the case when group-IV atoms substitute for $\mathrm{N}$ and form acceptors. The electronic structure of acceptors is more complex than that of donors. In zincblende materials, acceptor levels of group-IV atoms are threefold degenerate due to the cubic symmetry of the host. In GaN and AlN with wurtzite symmetry, the crystal field splits the triplet into a doublet and a singlet. Here, the energy of doublet-singlet splitting is denoted by $E_{\text {split }}$. In all cases considered here, the energies of doublets, $E_{\mathrm{DB}}$, are higher than those of singlets, $E_{S}$. For the neutral charge state of acceptors, the singlet is occupied by two electrons, and the doublet by three electrons. Both the energy levels and the splitting energies strongly depend on the impurity. The calculated values of $E_{\mathrm{DB}}$ for neutral acceptors are listed in Table I. We find that $\mathrm{C}$ is a shallow acceptor with $E_{\mathrm{DB}}=0.2 \mathrm{eV}$ in $\mathrm{GaN}$, and deeper $\left(E_{\mathrm{DB}}=0.5 \mathrm{eV}\right)$ and more localized in AlN. The doublet-singlet splitting $E_{\text {split }}$ is about $0.2 \mathrm{eV}$ in both materials. In contrast, both $\mathrm{Si}$ and $\mathrm{Ge}$ are deep acceptors. For $\mathrm{Si}(\mathrm{Ge})$ in $\mathrm{GaN}$ we find $E_{\mathrm{DB}}=1.2(1.35) \mathrm{eV}$ and $E_{\text {split }}=0.6$ (0.6) $\mathrm{eV}$. In AlN the binding energies are even higher, with $E_{\mathrm{DB}}=2.0 \mathrm{eV}$ and $E_{\text {split }}=0.65 \mathrm{eV}$ for $\mathrm{Si}_{\mathrm{N}}$, and $E_{\mathrm{DB}}=2.1 \mathrm{eV}$ and $E_{\text {split }}=0.65 \mathrm{eV}$ for $\mathrm{Ge}_{\mathrm{N}}$. From these results it follows that the incorporation of $\mathrm{Si}$ and $\mathrm{Ge}$ on the $\mathrm{N}$ sites does not result in $p$-type samples; this situation is dissimilar to that in, e.g., GaAs, where both $\mathrm{Si}$ and $\mathrm{Ge}$ are shallow acceptors.

According to our results, the energy levels of $\mathrm{Si}$ and $\mathrm{Ge}$ are similar, but they are qualitatively different from those of C. These differences are due to both different atomic energies of $p$ orbitals, which are about $1 \mathrm{eV}$ lower for $\mathrm{C}$ than for $\mathrm{Si}$ and $\mathrm{Ge}$, and differences in atomic radii, which affect both atomic relaxations and the energies of the impurity states.

\section{FORMATION ENERGIES AND COMPENSATION EFFECTS}

The concentration of an impurity at thermodynamic equilibrium is given by 


$$
\text { [conc }]=N_{\text {sites }} \exp \left(S_{\text {form }} / k_{B}-E_{\text {form }} / k_{B} T\right),
$$

where $N_{\text {sites }}$ (equal to 36 atoms in a 72-atom cell) is the concentration of atomic sites, and $S_{\text {form }}$ is its formation entropy, which is typically about $4 k_{B}-8 k_{B}$.

The formation energy of, e.g., $\mathrm{Si}$ in $\mathrm{GaN}$ in a charge state $q$ is

$$
E_{\text {form }}(q)=E_{\text {tot }}(q)-n_{\mathrm{Ga}} \mu_{\mathrm{Ga}}-n_{\mathrm{N}} \mu_{\mathrm{N}}-\mu_{\mathrm{Si}}+q E_{F},
$$

where $E_{\text {tot }}$ is the total energy of the supercell with the impurity, $n_{\mathrm{Ga}}$ and $n_{\mathrm{N}}$ are the numbers of $\mathrm{Ga}$ and $\mathrm{N}$ atoms in the supercell, $\mu$ is the chemical potential, and $E_{F}$ is the Fermi energy. The chemical potentials depend on the source of atoms involved in the process, and therefore on the actual experimental situation. At equilibrium,

$$
\mu_{\mathrm{Ga}}+\mu_{\mathrm{N}}=\mu_{\mathrm{GaN}}=\mu_{\mathrm{Ga}(\text { bulk })}+\mu_{\mathrm{N}_{2}}+\Delta H_{f}(\mathrm{GaN}),
$$

where $\Delta H_{f}$ is the heat of formation, which is negative for a stable compound. The highest possible concentration of a given dopant is obtained at the site of its lowest formation energy, see Eq. (2), and at the highest level of the chemical potential at the source of the dopant atoms. For $\mathrm{C}$ doping, we have assumed that the source is an elemental solid, i.e., graphite, which sets the correct upper bound for $\mu_{C}$. Consequently, the values of the formation energies of both $\mathrm{C}_{\mathrm{Ga}}$ and $\mathrm{C}_{\mathrm{N}}$ change by the value of the heat of formation of $\mathrm{GaN}$ between the two extremal conditions of growth, the Ga-rich and the N-rich limits. For $\mathrm{Si}$ and $\mathrm{Ge}$ doping, the possible formation of $\mathrm{Si}_{3} \mathrm{~N}_{4}$ and $\mathrm{Ge}_{3} \mathrm{~N}_{4}$ stable alloys lowers the upper bound of $\mu$ due to an additional constraint ${ }^{25}$ on the chemical potentials, e.g.:

$$
3 \mu_{\mathrm{Si}}+4 \mu_{\mathrm{N}}=\mu_{\mathrm{Si}_{3} \mathrm{~N}_{4}}=3 \mu_{\mathrm{Si}(\text { bulk })}+4 \mu_{\mathrm{N}_{2}}+\Delta H_{f}\left(\mathrm{Si}_{3} \mathrm{~N}_{4}\right) .
$$

By combining Eqs. (3)-(5) we find that the chemical potential of Si depends on the conditions of growth:

$$
\mu_{\mathrm{Si}}=\mu_{\mathrm{Si}(\text { bulk })}+(1 / 3) \Delta H_{f}\left(\mathrm{Si}_{3} \mathrm{~N}_{4}\right)-\frac{4}{3} \Delta H_{f}(\mathrm{GaN})
$$

for the Ga-rich limit, and

$$
\mu_{\mathrm{Si}}=\mu_{\mathrm{Si}(\text { bulk })}+\frac{1}{3} \Delta H_{f}\left(\mathrm{Si}_{3} \mathrm{~N}_{4}\right)
$$

for the N-rich limit. Therefore, for the N-rich case, the upper bound of $\mu_{\mathrm{Si}}$ is always reduced. This result is due to the fact that in the presence of excess nitrogen silicon atoms form the stable compound $\mathrm{Si}_{3} \mathrm{~N}_{4}$. The upper bound of $\mu_{\mathrm{Si}}$ is reduced form its bulk value also for the Ga-rich case [Eq. (7)], since

$$
\frac{1}{3} \Delta H_{f}\left(\mathrm{Si}_{3} \mathrm{~N}_{4}\right)-(4 / 3) \Delta H_{f}(\mathrm{GaN})<0 .
$$

However, for GaN:Ge, AlN:Si, and AlN:Ge in the cationrich limit, the relation analogous to (8) is not fulfilled, and the corresponding upper bounds are not reduced from their bulk values.

The calculated formation energies are given in Table III. ${ }^{26}$ As follows from the table, $\mathrm{C}$ is preferentially incorporated on the anion sublattice (except for the N-rich limit in AlN,
TABLE III. Calculated formation energies (in eV) of neutral substitutional impurities at cation-rich and N-rich conditions.

\begin{tabular}{lcc}
\hline \hline Dopant & $\begin{array}{c}\text { Cation-rich } \\
\text { GaN }\end{array}$ & N-rich \\
\hline $\mathrm{C}_{\mathrm{Ga}}$ & 5.7 & 4.0 \\
$\mathrm{C}_{\mathrm{N}}$ & 1.1 & 2.8 \\
$\mathrm{Si}_{\mathrm{Ga}}$ & 0.9 & 1.4 \\
$\mathrm{Si}_{\mathrm{N}}$ & 3.0 & 6.9 \\
$\mathrm{Ge}_{\mathrm{Ga}}$ & 2.3 & 2.2 \\
$\mathrm{Ge}_{\mathrm{N}}$ & 3.1 & 6.4 \\
& & \\
& $\mathrm{AlN}$ & \\
$\mathrm{C}_{\mathrm{Al}}$ & 7.7 & 3.4 \\
$\mathrm{C}_{\mathrm{N}}$ & 0.5 & 3.8 \\
$\mathrm{Si}_{\mathrm{Al}}$ & 3.5 & 2.3 \\
$\mathrm{Si}_{\mathrm{N}}$ & 2.6 & 10.0 \\
$\mathrm{Ge}_{\mathrm{Al}}$ & 5.1 & 2.4 \\
$\mathrm{Ge}_{\mathrm{N}}$ & 2.8 & 8.7 \\
\hline \hline
\end{tabular}

where the formation energies of $\mathrm{C}_{\mathrm{Al}}$ and $\mathrm{C}_{\mathrm{N}}$ are comparable), while both $\mathrm{Si}$ and $\mathrm{Ge}$ substitute for cations. These results are simply explained by the presence of residual strain energy when the mismatch between the host and the impurity atoms is large. In these cases, the excess strain energy is of the order of a few eV, as pointed out in Sec. III. However, as we show below, charge-transfer effects may overcome this effect.

Formation energies listed in Table III are those of neutral impurities. In the case of charged impurities, formation energies are in general reduced by electron transfer to/from the Fermi level according to Eq. (3). Since the energy gain may be of the order of the band gap, the formation of charged defects is of particular importance in wide-band-gap materials. (This situation is similar to that of native defects, discussed recently in Ref. 27.) For the dopants considered here, the electron transfer effects may overcame the strain-driven preference of the dopant atom $X$ to substitute for the component of the similar size, rendering formation energies of $X_{\text {cation }}$ and $X_{\mathrm{N}}$ close to each other. Consequently, a simultaneous incorporation of $X$ on both sublattices (i.e., selfcompensation) becomes possible. However, as we show below, the degree of self-compensation strongly depends on the conditions of growth, and may vary from none to total selfcompensation.

Defect concentrations are also very sensitive to the growth conditions, i.e., the temperature and the chemical potentials of the species involved. For this reason, and for the sake of transparency, we have performed calculations for a limited set of parameters, choosing various extremal conditions of growth in order to assess when an efficient and nonself-compensated doping is expected at all.

From Table III, for Si and $\mathrm{Ge}$ in the N-rich conditions of growth the difference of formation energies between $X_{\mathrm{N}}$ and $X_{\text {cation }}$ exceeds the value of the band gap. This holds for both $\mathrm{GaN}$ and AlN. Therefore, under these conditions, energy gain due to charge transfer from donor to acceptor cannot overcome strain-driven effects, and the concentration of $X_{\text {cation }}$ is always higher than that of $X_{\mathrm{N}}$, i.e., self- 
compensation is negligible. We observe, however, that in this limit $X_{\text {cation }}$ donors can still be compensated for by the dominant native acceptors, which are cation vacancies. ${ }^{27} \mathrm{In}$ particular, the calculated solubility limit of $\mathrm{Si}$ at $800{ }^{\circ} \mathrm{C}$ exceeds $10^{20} \mathrm{~cm}^{-3}$, but the degree of compensation by $V_{\mathrm{Ga}}$ is high, because formation of cation vacancies is an efficient process in the N-rich conditions. Analogous arguments hold for doping of GaN with carbon. From Table III it follows that under cation-rich conditions of growth, the formation energies of $\mathrm{C}_{\text {cation }}$ are larger than these of $\mathrm{C}_{\mathrm{N}}$ by more than the band gap. Thus, in this limit, carbon is a non-selfcompensated acceptor. However, $\mathrm{C}_{\mathrm{N}}$ may be compensated for by dominant native donors, which are nitrogen vacancies and/or Ga interstitials. ${ }^{27,28}$

On the other hand, under the opposite conditions of growth (i.e., the cation-rich limit for $\mathrm{Si}$ and $\mathrm{Ge}$, and the Nrich limit for $\mathrm{C}$ ), self-compensation may play a dominant role. In the case of GaN:C, GaN:Ge, AlN:C, AlN:Si, and AlN:Ge, a value of the Fermi level exists for which $E_{\text {form }}\left(X_{\text {cation }}^{+}\right)=E_{\text {form }}\left(X_{\mathrm{N}}^{-}\right)$, at which point the concentrations of $X_{\text {cation }}^{+}$and $X_{\mathrm{N}}^{-}$should be the same. This implies that under equilibrium conditions doping with group-IV atoms should lead to self-compensation and pinning of the Fermi level. For $\mathrm{Si}$ in $\mathrm{GaN}$, however, self-compensation effects are not expected except in the very Ga-rich limit. In practice, kinetic effects may limit dopant incorporation, and thus allow for reasonably effective doping.

We end this section by comparing our results with other theoretical calculations for dopants in GaN. In Ref. 29, somewhat higher formation energies for both $\mathrm{C}_{\mathrm{Ga}}, 6.5 \mathrm{eV}$, and $\mathrm{Si}_{\mathrm{Ga}}, 1.2 \mathrm{eV}$, were obtained, but they are still in a satisfactory agreement with the present work. ${ }^{26}$ Fiorentini et al. ${ }^{30}$ studied potential acceptors in $\mathrm{GaN}$. Considering $\mathrm{C}_{\mathrm{N}}$, they find that its acceptor level, $E_{\text {acc }}=0.65 \mathrm{eV}$, is deeper than what is obtained here, and the formation energy $E_{\text {form }}=4.2$ $\mathrm{eV}$ is higher than our value of $2.8 \mathrm{eV}$. Also, $\mathrm{Si}_{\mathrm{N}}$ with $E_{\text {acc }}=1.97 \mathrm{eV}$, is predicted to be deeper by about $0.8 \mathrm{eV}$ than what we find, while $E_{\text {form }}=5.5 \mathrm{eV}$ at N-rich conditions is lower than our value of $6.9 \mathrm{eV}$. A comparison of Table 1 from Ref. 30 with Table I of this work shows that the relaxation effects predicted here are somewhat larger for both dopants; e.g., for $\mathrm{Si}_{\mathrm{N}}$, Fiorentini et al. found $\Delta b=12.0 \%$, and $E_{\text {rel }}=3.4$, while our respective values are $13.6 \%$ and 3.9 $\mathrm{eV}$. These differences may be due to a larger unit cell used in the present work, which allows for a greater magnitude of atomic relaxations, and possibly differences in the atomic pseudopotentials. $\mathrm{Si}_{\text {cation }}$ donors were recently investigated by Mattila and Nieminen. ${ }^{31}$ They considered cubic GaN and AlN, which makes the comparison less direct. However, their results for $\mathrm{GaN}: \mathrm{Si}$ are close to ours: $\mathrm{Si}_{\mathrm{Ga}}$ is a shallow donor, and its $E_{\text {form }}=1.6 \mathrm{eV}$ is in an acceptable agreement with our value. The discrepancies are somewhat larger for $\mathrm{Si}$ in AlN. In particular, their $E_{\text {form }}$ is lower than our value by about $1.0 \mathrm{eV}$. Moreover, they find $\mathrm{Si}$ to be a deep donor. The inward relaxation of nearest neighbors for $\mathrm{Si}^{+}$is similar to that found here. The pronounced asymmetry of the atomic relaxation around $\mathrm{Si}_{\mathrm{Al}}^{-}$, i.e., the elongation of the bond with one nearest neighbor by $12 \%$, and the shortening of the three remaining bonds, may be a precursor of the stabilization of
TABLE IV. Binding energies $E_{\text {pair }}$ and short-range interaction energies $E_{\mathrm{sr}}$ for nearest-neighbor impurity pairs.

\begin{tabular}{lcc}
\hline \hline & $E_{\text {pair }}(\mathrm{eV})$ & $E_{\text {sr }}(\mathrm{eV})$ \\
& $\mathrm{GaN}$ & \\
\hline $\mathrm{C}-\mathrm{C}$ & -1.1 & -0.2 \\
$\mathrm{Si}-\mathrm{Si}$ & -0.8 & -0.15 \\
$\mathrm{Ge}-\mathrm{Ge}$ & -1.1 & -0.4 \\
& & \\
\hline $\mathrm{C}-\mathrm{C}$ & $\mathrm{AlN}$ & \\
$\mathrm{Si}-\mathrm{Si}$ & -1.4 & -0.5 \\
$\mathrm{Ge}-\mathrm{Ge}$ & -1.3 & -0.7 \\
\hline \hline
\end{tabular}

the $D X$ configuration that we find in the wurtzite AlN; however, $D X$-like states with large lattice relaxations were not analyzed in Ref. 31.

\section{FORMATION OF NEAREST-NEIGHBOR DONOR-ACCEPTOR PAIRS}

The degree of self-compensation may be further increased by the formation of donor-acceptor nearest-neighbor $(\mathrm{NN})$ pairs. Formation of such pairs is an independent mode of incorporation of impurity atoms. In a simple picture which neglects the coupling between second-neighbor or more distant pairs, the total concentration of an impurity $X$ is the sum of $\left[X_{\text {cation }}\right],\left[X_{\mathrm{N}}\right]$, and $[X X]$. The formation of NN pairs increases both the solubility (as was discussed for $\mathrm{Si}$ in GaAs in Ref. 32) and the degree of self-compensation, but does not change the concentrations of conduction electrons or holes. However, the presence of the pairs may significantly lower carrier mobility since they introduce an additional scattering channel.

As the result of electron transfer from donors to acceptors in the pairs, both $X_{\text {cation }}$ donors and $X_{\mathrm{N}}$ acceptors are ionized. According to our results, the binding energy of a secondneighbor, or a more distant, pair is essentially the Coulomb interaction between two point charges embedded in the medium with the appropriate dielectric constant. However, the binding in a NN pair deviates significantly from the purely Coulombic character. This is not surprising, since the picture of interacting point charges should not hold for a NN pair, given the finite extent of the impurity wave functions. We define the binding energy of a NN pair as

$$
E_{\text {pair }}=E_{\mathrm{NN}}-E_{\infty}
$$

where $E_{\mathrm{NN}}$ and $\mathrm{E}_{\infty}$ are the total energies of a NN pair and of a distant pair, respectively. The additional short-range interaction is defined as

$$
E_{\mathrm{sr}}=E_{\text {pair }}-E_{\text {Coul }}^{\mathrm{NN}},
$$

where $E_{\mathrm{Coul}}^{\mathrm{NN}}$ is the Coulomb energy of a pair of point charges separated by the theoretical NN distance.

The calculated values of $E_{\text {pair }}$ and $E_{\mathrm{sr}}$, corrected for the spurious interaction between pairs in different supercells, are given in Table IV. We have only investigated the NN pairs 
oriented along the $c$ axis. The pairing energies are about 1 $\mathrm{eV}$, which is higher than the $\mathrm{Si}-\mathrm{Si}$ binding energy in GaAs, ${ }^{32,33}$ due in part to a smaller NN distance and a smaller dielectric constant. The additional short-range interaction $E_{\text {sr }}$ is always attractive. It ranges from $-0.15 \mathrm{eV}$ for a $\mathrm{Si}-\mathrm{Si}$ pair in $\mathrm{GaN}$ to $-1.0 \mathrm{eV}$ for a Ge-Ge pair in AlN (which is about $60 \%$ of the binding energy). We also see that $E_{\mathrm{sr}}$ is systematically larger in AlN than in $\mathrm{GaN}$ by $0.3-0.6 \mathrm{eV}$. The attractive character of the short-range coupling is in part due to the release of excess strain, which is more efficient for NN than for more distant pairs.

The formation energy of a NN pair is

$$
E_{\text {form }}(X X)=E_{\text {form }}\left(X_{\text {cation }}^{+}\right)+E_{\text {form }}\left(X_{\mathrm{N}}^{-}\right)+E_{\text {pair }},
$$

which is independent of the Fermi level $E_{F}$ for neutral pairs, i.e., for $E_{A}<E_{F}<E_{D}$. The concentration of $\mathrm{NN}$ pairs is given by Eq. (2), with $\mathrm{N}_{\text {sites }}$ four times larger than that for substitutional impurities. Thus $[X X]$ is comparable to $\left[X_{\text {cation }}\right]$ or $\left[X_{\mathrm{N}}\right]$ only $\left|E_{\text {pair }}\right|$ $\geqslant \max \left[E_{\text {form }}\left(X_{\text {cation }}^{+}\right), E_{\text {form }}\left(X_{\mathrm{N}}^{-}\right)\right]$.

The concentration of NN pairs may be calculated based on the results from Tables I, III, and IV. Due to the fact that $\mathrm{C}$ does not form a stable compound with $\mathrm{N}$, the concentration of C-C pairs is independent of the conditions of growth. We find that pairing of $\mathrm{C}$ is negligible in $\mathrm{GaN}$, but can be important in AlN, especially under N-rich conditions. For both $\mathrm{Si}$ and $\mathrm{Ge}$ in both $\mathrm{GaN}$ and AlN under the N-rich conditions, we find that the concentration of NN pairs is negligibly small compared with the concentrations of the isolated impurities. However, the pairing may be important in the cation-rich limit. In particular, for $\mathrm{Si}$ in $\mathrm{GaN}$, the concentration of $\mathrm{NN}$ pairs is comparable to that of $\left[\mathrm{Si}_{\mathrm{Ga}}\right]$ and $\left[\mathrm{Si}_{\mathrm{N}}\right]$ in the Ga-rich limit. In AlN:Si, most of $\mathrm{Si}$ impurities form pairs under the Al-rich conditions. Finally, in the case of Ge in $\mathrm{GaN},[\mathrm{Ge}-\mathrm{Ge}]$ is smaller by one order of magnitude that $[\mathrm{Ge}$ $\mathrm{Ga}]$ and $\left[\mathrm{Ge}_{\mathrm{N}}\right]$, but in AlN the concentration of Ge pairs is close to that of isolated Ge impurities in the Al-rich limit.

The electronic structure of the nearest-neighbor $X_{\text {cation }}^{+}$ $-X_{\mathrm{N}}^{-}$pairs is similar to that of distant $X_{\text {cation }^{+}}$and $X_{\mathrm{N}}^{-}$impurities, the main modification being a factor of 2 increase in the doublet-singlet splittings. More specifically, in $\mathrm{GaN} E_{\text {split }}$ is about $0.4,1.0$, and $1.05 \mathrm{eV}$ for $\mathrm{C}-\mathrm{C}, \mathrm{Si}-\mathrm{Si}$, and $\mathrm{Ge}-\mathrm{Ge}$ pairs, respectively. For AlN, the splitting increases to about $0.7,1.4$, and $1.35 \mathrm{eV}$ for $\mathrm{C}-\mathrm{C}, \mathrm{Si}-\mathrm{Si}$, and $\mathrm{Ge}-\mathrm{Ge}$ pairs, respectively. This effect is due to the close proximity of the $X_{\text {cation }}$ donor, located along the $c$-axis relative to the acceptor $X_{\mathrm{N}}$. The presence of the donor enhances the nonequivalence between the $z$-symmetry singlet, pulled down by the donor potential, and the $(x, y)$-symmetry doublet.

\section{SUMMARY}

In summary, we have investigated doping properties of $\mathrm{C}$, $\mathrm{Si}$, and $\mathrm{Ge}$ in wurtzite $\mathrm{GaN}$, AlN, and $\mathrm{Al}_{x} \mathrm{Ga}_{1-x} \mathrm{~N}$ random alloys by quantum molecular dynamics. Incorporation of impurities on both cation and $\mathrm{N}$ sublattices was considered. We have analyzed effects that could potentially limit the doping efficiency, namely; (i) stabilization of donors in $D X$ configurations, (ii) self-compensation due to the simultaneous incorporation of impurities on both sublattices, and (iii) the formation of nearest-neighbor donor-acceptor pairs. In cases when the atomic radii of the impurity and of the substituted host atom are substantially different (e.g., $\mathrm{C}_{\mathrm{Ga}}$, or $\mathrm{Si}_{\mathrm{N}}$ ), the effects of atomic relaxations around the impurities and the induced strain strongly affect both the impurity levels and the formation energies. However, the strain-driven preference to substitute for the host atom with a similar size may be overcome by the energy gain due to electron transfer between a donor and an acceptor. If this is the case, doping at equilibrium conditions should lead to Fermi-level pinning, at which point the formation energy of the donor becomes equal to that of the acceptor, and thus total selfcompensation takes place.

We find the following.

(i) $\mathrm{C}_{\mathrm{N}}$ is a promising acceptor, as it is somewhat more shallow than the commonly used Mg (Refs. 34 and 4) and it should have a significantly greater solubility than atomic $\mathrm{Mg}$. However, the presence of $\mathrm{H}$ aids in incorporation of $\mathrm{Mg},{ }^{35,36}$ although in this case post-growth activation of $\mathrm{Mg}$ is required.

(ii) Negligible self-compensation and pairing effects are expected for $\mathrm{Al}_{x} \mathrm{Ga}_{1-x} \mathrm{~N}$ alloys in the Ga-rich limit, i.e., for compositions $0<x<0.4$.

(iii) In $\mathrm{Al}_{x} \mathrm{Ga}_{1-x} \mathrm{~N}$ alloys with composition $x<0.60$ grown in the $\mathrm{N}$-rich conditions, $\mathrm{Si}_{\text {cation }}$ is an excellent effective-mass donor; neither self-compensation nor the formation of NN pairs is expected. For alloys with compositions higher than about 0.60 , the $D X_{1}^{-}$configuration is stable, which quenches the doping efficiency.

(iv) For the N-rich conditions of growth, $\mathrm{Ge}_{\text {cation }}$ is an excellent effective-mass donor in $\mathrm{Al}_{x} \mathrm{Ga}_{1-x} \mathrm{~N}$ for compositions lower than about 0.3 . For $x>0.3$, the doping efficiency is quenched due to the stabilization of the $D X_{1}^{-}$state. Furthermore, appreciable self-compensation and pairing are expected.

Samples grown under the conditions opposite to those specified above are expected to be self-compensated to a high degree. Our results for $\mathrm{Si}$ and $\mathrm{Ge}$ indicate that the two common $n$-type dopants in $\mathrm{GaN}, \mathrm{Si}$ and $\mathrm{Ge}$, are not shallow donors in AlN and Al-rich alloys; this opens the question of finding not only efficient acceptors, but also appropriate donors for Al-rich materials.

Note added. A recent paper (Ref. 37) also investigated the stability of $D X$ states of $\mathrm{Si}$ and Ge dopants in wurtzite GaN and AlN. This paper analyzed configurations with the broken bond parallel to the $c$ axis (calling it $\gamma$-BB, an analog of our $D X_{1}$ state), and along one of the three remaining bonds (called $\alpha$-BB, an analog of our $D X_{2}$ state). The $\gamma$-BB configuration considered in Ref. 37 had a displaced $\mathrm{Si}$ or $\mathrm{Ge}$ impurity atom and an almost nondisplaced host $\mathrm{N}$ atom. It was found unstable for both $\mathrm{Si}$ and $\mathrm{Ge}$ in $\mathrm{Al}_{x} \mathrm{Ga}_{1-x} \mathrm{~N}$ alloys. This is in agreement with our results. The configurations $D X_{1}$ that we have considered have the reverse displacements, with the impurity atom almost non-displaced and the host $\mathrm{N}^{*}$ strongly displaced along the $c$ axis (see Fig. 1 and Sec. IV). This variant turned out to be metastable (for Ge in $\mathrm{GaN}$ ), or even stable (for both $\mathrm{Si}$ and Ge in AlN, see Table II).

Turning to the $\alpha$-BB $\left(D X_{2}\right)$ variant, both we and Ref. 37 find it unstable for $\mathrm{Ge}$ in $\mathrm{Al}_{x} \mathrm{Ga}_{1-x} \mathrm{~N}$ for all compositions. However, for $\mathrm{Si}$ the agreement is less satisfactory: Ref. 37 
found that this variant is metastable in $\mathrm{GaN}$, and stable in AlN. We find that this variant is much less stable: in GaN it is unstable, and in AlN it is less stable than $D X_{1}$ by $0.95 \mathrm{eV}$. Our results imply that the shallow-deep transition occurs at a higher Al concentration (cf. Sec. IV H), which appears to be supported by recent experiments. ${ }^{5}$ Finally, an impurity may have several local minima with large lattice relaxations, as was found for $\mathrm{CdTe}: \mathrm{Cl}^{38}$ but the configuration shown in Fig. 3(d) of Ref. 37 is too sketchy to allow for a detailed comparison with our results.

\section{ACKNOWLEDGMENTS}

This work was supported in part by Grant Nos. ONR N00014-92-J-1477, NSF DMR 9408437, and KBN 2-P03B178-10. The authors would like to thank Dr. Z. Wilamowski and Dr. Q.-M. Zhang for useful discussions.
${ }^{1}$ R. F. Davis, Physica B 185, 1 (1993).

${ }^{2}$ H. Morkoc et al., J. Appl. Phys. 76, 1363 (1994).

${ }^{3}$ S. Nakamura, T. Mukai, and M. Senoh, J. Appl. Phys. 31, 2883 (1992).

${ }^{4}$ S. Fischer, C. Wetzel, E. E. Haller, and B. K. Meyer, Appl. Phys. Lett. 67, 1298 (1995)

${ }^{5}$ M. Bremser and R. F. Davis (unpublished).

${ }^{6}$ C. R. Abernathy, J. D. MacKenzie, S. J. Pearton, and W. S. Hobson, Appl. Phys. Lett. 66, 1969 (1995).

${ }^{7}$ L. B. Rowland, K. Doverspike, and D. K. Gaskill, Appl. Phys. Lett. 66, 1495 (1996).

${ }^{8}$ C. Wetzel, A. L. Chen, T. Suski, J. W. Ager III, and W. Walukiewicz, Physica Status Solidi B 198, 243 (1996).

${ }^{9}$ X. Zhang, P. Kung, A. Saxler, D. Walker, T. C. Wang, and M. Razeghi, Appl. Phys. Lett. 67, 1745 (1995).

${ }^{10}$ G.-C. Yi and B. Wessels, Appl. Phys. Lett. 70, 357 (1997).

${ }^{11}$ R. Car and M. Parrinello, Phys. Rev. Lett. 55, 2471 (1985).

${ }^{12}$ C. Wang, Q.-M. Zhang, and J. Bernholc, Phys. Rev. Lett. 69, 3789 (1992).

${ }^{13} \mathrm{G}$. Li and S. Rabii (unpublished).

${ }^{14}$ X. Gonze, R. Stumpf, and M. Scheffler, Phys. Rev. B 44, 8503 (1991).

${ }^{15}$ V. Fiorentini, M. Methfessel, and M. Scheffler, Phys. Rev. B 47, 13353 (1993).

${ }^{16}$ M. Buongionro Nardelli, K. Rapcewicz, and J. Bernholc, Phys. Rev. B 55, R7323 (1997).

${ }^{17}$ P. N. Keating, Phys. Rev. 145, 637 (1966); R. M. Martin, Phys. Rev. B 1, 4005 (1970). The bond-stretching and -bending constants were obtained by scaling the values used by A. B. Chen and A. Sher, ibid. 32, 3695 (1985) for other III-V semiconductors. For GaN (AlN) we use $\alpha=80$ (89) $\mathrm{N} / \mathrm{m}$, and $\beta=12$ (13) $\mathrm{N} / \mathrm{m}$.

${ }^{18}$ D. J. Chadi and K. J. Chang, Phys. Rev. Lett. 61, 873 (1988).

${ }^{19}$ The effects of the band structure and chemical bonding on the stability of $D X$ states in GaAs and InP were discussed by B. H. Cheong and K. J. Chang, Phys. Rev. Lett. 71, 4354 (1993).

${ }^{20}$ T. M. Schmidt, A. Fazzio, and M. J. Caldas, Phys. Rev. B 53, 1315 (1996).

${ }^{21}$ P. Boguslawski and I. Gorczyca, Semicond. Sci. Technol. 9, 2169 (1994).

${ }^{22}$ Strictly speaking, even in case (c) of Fig. 2, a sample may be conducting at finite temperatures. The progressive decrease of the energy of the $D X$ state with the alloy composition investigated in this section results in an increasing activation energy of the conduction electrons. In addition, the conductivity may be due to persistent effects discussed in the following.

${ }^{23} \mathrm{We}$ note that even in the absence of the impurity, total energies of supercells with different distributions of cations are in general different. Thus, to obtain energy of $D X$ state relative to the substitutional one, one has to analyze both states for each distribution of cations.

${ }^{24}$ Z. Wilamowski, J. Kossut, W. Jantsch, and G. Ostermayer, Semicond. Sci. Technol. 6, B38 (1991).

${ }^{25}$ C. G. Van De Walle, D. B. Laks, G. F. Neumark, and S. T. Pantelides, Phys. Rev. B 47, 9425 (1993).

${ }^{26}$ The results of Table III were obtained using theoretical values of $\Delta H_{f}$ of the considered materials, except for $\mathrm{Si}_{3} \mathrm{~N}_{4}$ and $\mathrm{Ge}_{3} \mathrm{~N}_{4}$. Cohesive energies of these two compounds were not computed. Instead, we used values based on experiment; to correct for the usual overestimation of formation energies in local-density theory, we increased the experimental values by $20 \%$, which is the average discrepancy between the computed and experimental values for bulk $\mathrm{Si}, \mathrm{Ge}$, and $\mathrm{N}_{2}$. This correction of $\Delta H_{f}$ results in a $0.5-\mathrm{eV}$ increase of $E_{\text {form }}$ for $\mathrm{Si}$ in both GaN and AlN in the $N$-rich limit [Eq. (7)]. The corresponding increase in the formation energy for $\mathrm{Ge}$ is $0.3 \mathrm{eV}$.

${ }^{27}$ P. Boguslawski, E. L. Briggs, and J. Bernholc, Phys. Rev. B 51, 17255 (1995).

${ }^{28}$ P. Perlin, T. Suski, H. Teisseyre, M. Leszczynski, I. Grzegory, J. Jun, S. Porowski, P. Boguslawski, J. Bernholc, J. C. Chervin, A. Polian, and T. D. Moustakas, Phys. Rev. Lett. 75, 296 (1995).

${ }^{29} \mathrm{~J}$. Neugebauer and C. Van de Walle, in Proceedings of the $22 \mathrm{nd}$ International Conference on the Physics of Semiconductors, Vancouver, 1994, edited by D. J. Lockwood (World Scientific, Singapore, 1995), p. 2327.

${ }^{30}$ F. Fiorentini, F. Bernardini, A. Bosin, and D. Vanderbilt, in Proceedings of the 23rd International Conference on the Physics of Semiconductors, Berlin, 1996, edited by M. Scheffler and R. Zimmerman (World Scientific, Singapore, 1996), p. 2877.

${ }^{31}$ T. Mattila and R. M. Nieminen, Phys. Rev. B 55, 9571 (1997).

${ }^{32}$ J. E. Northrup and S. B. Zhang, Phys. Rev. B 47, 6791 (1993).

${ }^{33}$ B. Chen, Q.-M. Zhang, and J. Bernholc, Phys. Rev. B 49, 2985 (1994).

${ }^{34}$ P. Boguslawski, E. L. Briggs, and J. Bernholc, Appl. Phys. Lett. 69, 233 (1996).

${ }^{35}$ J. A. Van Vechten, J. D. Zook, R. D. Hornig, and B. Goldenberg, Jpn. J. Appl. Phys. 31, 3662 (1992).

${ }^{36}$ J. Neugebauer and C. G. Van de Walle, Appl. Phys. Lett. 68, 1829 (1996).

${ }^{37}$ C. H. Park and D. J. Chadi, Phys. Rev. B 55, 12995 (1997).

${ }^{38}$ C. H. Park and D. J. Chadi, Appl. Phys. Lett. 66, 3167 (1995). 